\title{
Non-Contact Simultaneous Measurements of Electrocardiogram and Respiratory Movements Using Capacitive Sheet Electrodes
}

\author{
Mayuko TAKano,,$\#$ Shinsuke YAmagishi, ${ }^{* *}$ Takao Ohmuta, ${ }^{* * *}$ Yutaka FuKuoKa, ${ }^{\dagger}$ Akinori Ueno ${ }^{\dagger \dagger}, \#$
}

\begin{abstract}
In this study, we improved upon a high-input impedance monitoring device comprising capacitive sheet electrodes placed under a bed sheet, aiming to detect all ECG waves including T waves. To accomplish this, we widened the passbands for ECG and respiratory movement (RM) signals. However, because this widening may lower noise tolerance, we substituted conventional front-end buffers with buffers able to discharge DC potential. Further, the backside of the modified electrode was doubly shielded by using the electric potential of the buffer's output and ground. We also introduced a virtual midpoint circuit to realize stable ECG and independent RM measurements of the chest and abdomen with the goal to detect obstructive sleep apnea in future applications. We tested our device on seven healthy adults during a six-hour sleep experiment. In all subjects, we detected T waves and observed ECG and RM signals in supine and lateral positions. In experiments in which respiratory rate was changed at regular intervals, the RM signal was synchronized with the simultaneously measured reference signal. The sensitivity and accuracy of $\mathrm{R}$ waves averaged over all subjects were $93.2 \%$ and $92.9 \%$, respectively. Both averaged sensitivity and accuracy for $\mathrm{T}$ waves were $94.5 \%$, while averaged accuracy of chest and abdominal RM was $70.9 \%$ and $74.0 \%$, respectively. The results for ECG signals indicate that our improved system is applicable to unconstrained monitoring of vital signs in adults. Although there is still room for improvement in RM measurements, the results suggest that our system may also be used to detect variations in breathing.
\end{abstract}

Keywords: non-contact, capacitive sheet electrode, electrocardiogram-respiratory movement measurement device, vitals monitoring.

Adv Biomed Eng. 6: pp. 28-36, 2017.

\section{Introduction}

Due to population aging in Japan, China and several other countries [1], a shortage of available beds in hospitals and increased spending on healthcare are predicted to become severe problems. The demand for residential monitoring systems to monitor vital signs of individuals is expected to increase substantially on a global scale. A method that does not require direct contact of electrodes and probes with the skin is a highly desirable goal, as such monitoring is expected to continue for weeks or more.

Numerous methods that do not require direct contact with the skin have been proposed for monitoring heart rates and respiration rates in patients, including methods using microwaves [2,

This study was presented at the Symposium on Biomedical Engineering 2016, Asahikawa, September, 2016.

Received on July 24, 2016; revised on November 10, 2016 and December 20, 2016; accepted on December 26, 2016.

*Master's Program in Electrical and Electronic Engineering, Tokyo Denki University, Tokyo, Japan.

** Master's Program in Electrical Engineering, Kogakuin University, Tokyo, Japan.

**** Department of Clinical Engineering, Faculty of Medical Engineering, Suzuka University of Medical Science, Suzuka, Japan.

${ }^{\dagger}$ Department of Electrical Engineering, Faculty of Engineering, Kogakuin University, Tokyo, Japan.

${ }_{\dagger \dagger}$ Department of Electrical and Electronic Engineering, School of Engineering, Tokyo Denki University, Tokyo, Japan.

\# 5 Senju-Asahi-cho, Adachi-ku, Tokyo 120-8551, Japan.

E-mail:16kmj27@ms.dendai.ac.jp, ueno@mail.dendai.ac.jp
3], ultrasonic waves [4], and magnetism [5]. Ishijima [6] and Park et al. [7] proposed methods for simultaneous measurement of ECG and respiratory movement (RM) signals using textile electrodes. However, their ECG measurements required direct contact with the skin. Although Lee et al. [8] proposed a non-contact measurement system of ECG signals using capacitive-coupling textile electrodes, they did not measure RM signals. In addition, coverage of $\mathrm{T}$ waves and QT intervals is yet to be evaluated. Yama and Ueno $[9,10]$ proposed a method for simultaneous measurement of ECG and RM signals in infants using capacitively coupled electrodes, but verification by continuous measurements over long periods of time was not performed, due to frequent interruption for feeding and diaper changing. Peltokangas et al. [11] proposed long-term night-time measurements using textile electrodes, although their ECG measurements also required direct contact with the skin.

To our knowledge, no system for simultaneous measurement of ECG and RM signals in adults without direct skin contact has been developed. The system of Yama and Ueno [10] noted above was intended for infants and allowed measurements through underwear and diapers, using electro-conductive fabric electrodes installed underneath commercially available bed sheets. The risk of bedsores arising from long-term use of such electrodes is low. Thus, there is potential for use in monitoring vital signs of adult patients at home. Use of this system in such setting requires changes of various parameters specific to adult application and verification of effective continuous monitoring over extended periods.

More specifically, the frequency band for the ECG detector of the system described previously [10] was set at 5 to $40 \mathrm{~Hz}$ to 
stabilize the baseline of the output signal. As a result, the ECG signals lacked the components in the 0.53 to $5 \mathrm{~Hz}$ range required for proper monitoring of ECG signals. Furthermore, the frequency band for the respiration detector was set at 0.1 to $1 \mathrm{~Hz}$, which is higher than that for adults. In addition, the conventional capacitive electrode used by Yama and Ueno [10] will probably accumulate static charges on the coupling part, thereby releasing saturated voltage levels after the subject changes posture. In capacitive ECG measurements through clothing, input impedance for the front-end voltage follower is usually set at values substantially higher than that for commercial ECG devices, thus incurring a long time for restoring to baseline after saturation. Therefore, simple bandwidth extension to lower frequencies (such as to $0.53 \mathrm{~Hz}$ ) would result in reduced ECG coverage due to the prolonged restoration time.

In this study, we used stabilization technology to improve the aforementioned capacitive in-bed cardiopulmonary monitor, with the aim to detect low-frequency components of ECG and $\mathrm{RM}$ signals in adults without coverage reduction. To evaluate the performance of our improved monitor, we conducted overnight experiments in seven subjects.

\section{Measurement system}

\subsection{Sheeted fabric electrode}

Figure 1 shows a cross-sectional diagram of the textile electrode used in our experiments and the connection to a front-end voltage follower. We used a double-shield electrode consisting of five layers to improve tolerance against disturbance [12]. The first layer is a sensing layer, the fifth layer is a ground plane, and the output from the front-end voltage follower is fed back to the third layer to configure a driven shield. We expected the driven shield to suppress current leakage from the sensing electrode to the fifth layer so as to improve the signal-to-noise ratio (SNR).

Moreover, the driven shield functions as a shield when disturbances occur, because the output impedance of the connected voltage follower is small. Studies of an in-vehicle ECG device have shown that tolerance against vibratory and electro-magnetic disturbances is improved using such a doubly shielded electrode (as compared to a single layer case) $[12,13]$.

Figure 2 shows the layout of electrodes and a block diagram of the proposed measurement system. The electrodes were placed under a bed sheet. We adopted the V-shape to prevent the individual's hands from touching the lower electrodes [14]. The hands are deemed to have equal potential as the arms. Accordingly, the amplitude of ECG signals may decrease when a hand touches the lower electrode.

\subsection{Front-end buffer circuit}

From our proposed layout, the electrodes coupled with the body through clothing are considered capacitive coupling with capacitance $C_{c p l}[\mathrm{~F}]$. Direct voltage $V_{c p l}=Q_{-} c p l / C_{c p l}$ occurs and becomes an artifact when electric charge $Q_{c p l}[\mathrm{C}]$ is accumulated in the coupling part due to friction. In a conventional voltage follower, the baseline restoration time is proportional to $1 /\left(2 \pi C_{c p l} R_{i n}\right)$, as determined by capacitance $C_{c p l}$ and the input resistance $R_{i n}$ of the operational amplifier.

With a small $C_{c p l}$; for example $10 \mathrm{pF}$, in a potential measure-

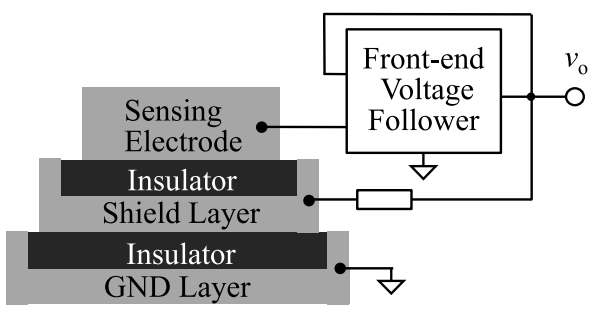

Fig. 1 Cross-sectional diagram of the electrode and connection to a front-end voltage follower. The sensing electrode is doubly shielded by the third and fifth layers. The output of the voltage follower is fed back to the third layer. The fifth layer serves as a ground plane.

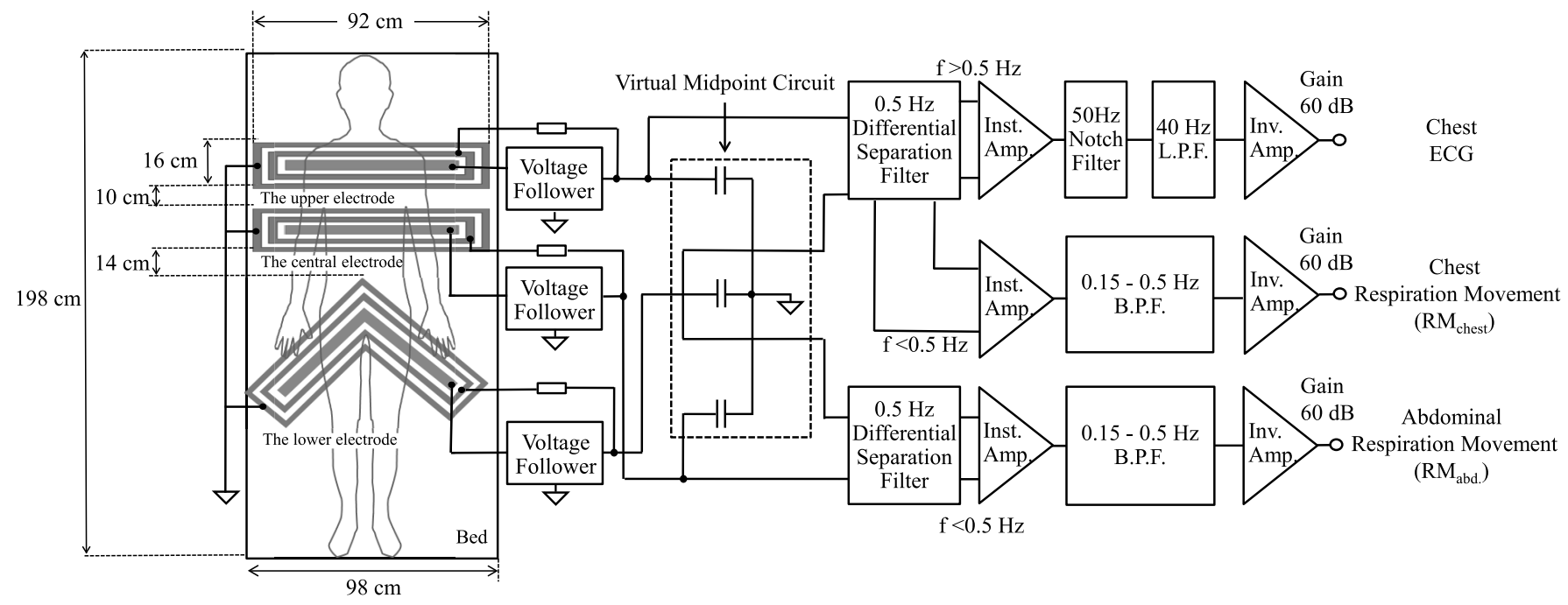

Fig. 2 Electrode layout and block diagram of the proposed measuring system. Electrodes are placed under a bed sheet at the upper back, waist, and buttock positions [14]. Our system consists of a front-end voltage follower with a bootstrap, a virtual midpoint circuit, differential separation filters, instrumentation amplifiers, filters, and amplifiers with a gain of $60 \mathrm{~dB}$. The frequency band for ECG signals is set at 0.5 to $40 \mathrm{~Hz}$, whereas the frequency band for RM signals is set at 0.15 to $0.5 \mathrm{~Hz}$. 
ment using capacitive coupling, the reactance in the coupling part for a frequency band between 0.5 and $40 \mathrm{~Hz}$ is $31 \mathrm{G}-400 \mathrm{M} \Omega$. Therefore, an operational amplifier IC with a large $R_{\text {in }}$ (for example, a nominal $1 \mathrm{~T} \Omega$ ) is often used to suppress the voltage loss in the coupling part. As a result, the time constant of the electric discharge increases (for example, $10 \mathrm{~s}$ when $C_{c p l}=10 \mathrm{pF}$ and $R_{\text {in }}=1 \mathrm{~T} \Omega$ ), and it takes time to recover to the baseline after natural body movements.

In this study, we focused on the fact that the detection signal is an AC signal, whereas the above discharge phenomenon has galvanic behavior. We therefore replaced the conventional voltage follower circuit with another voltage follower with a bootstrap, as illustrated in Fig. 3. In Fig. 3, a path between Q and O is considered open-circuit for DC signal because it cannot pass through capacitor $C_{f}$. Therefore, the input resistance of the circuit for the DC signal becomes $R_{b 1}+R_{b 2}$. Input impedance $Z_{i n}$ of the circuit for an AC signal is given as follows [15]:

$$
Z_{i n}=R_{b 1}+R_{b 2}+j 2 \pi f C_{f} R_{b 1} R_{b 2}
$$

At frequency $f$ within the band of the detection signal, if $1 /$ $\left(2 \pi f C_{f}\right) \ll R_{b 2}$, a voltage loss at the path between Q and $\mathrm{O}$ is closer to $0 \mathrm{~V}$, and the voltage of point $\mathrm{Q}$ equals $v_{o}$. Since the portion enclosed by dashed lines in Fig. $\mathbf{3}$ is a voltage follower $\left(v_{o}=v_{i}\right)$, the potential difference between points $\mathrm{P}$ and $\mathrm{Q}$ becomes nearly $0 \mathrm{~V}$. Then, the electric current between points $\mathrm{P}$ and $\mathrm{Q}$ is negligible small, and input impedance for the AC signal increases to ultra-high level. According to Equation (1), the baseline restoration time is proportional to $1 /\left\{2 \pi C_{c p l}\left(R_{b 1}+R_{b 2}\right)\right\}$. We therefore expect that this replacement contributes to a shorter baseline restoration time by choosing modest values for $R_{b 1}+R_{b 2}$, and a large value for $C_{f}$.

\subsection{Circuits for signal detection}

For the measuring device used to measure ECG and RM signals described by Yama and Ueno [10], the lower electrode was used as ground and the potential difference between the upper and central electrodes was amplified. Therefore, it was impossible to simultaneously acquire ECG and RM with large amplitudes. Furthermore, chest and abdominal RM signals could not be detected independently by their methods [10].

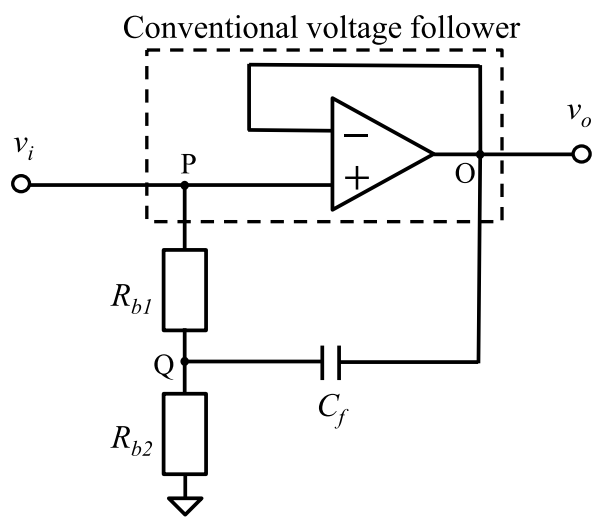

Fig. 3 Voltage follower with a bootstrap. The bootstrap circuit is expected to contribute to shortening the baseline restoration time, because the bootstrap circuit maintains a high impedance at the signal frequency band while lowering DC signal resistance.
We address these problems by detecting the electric potential of the three electrodes independently by introducing a virtual midpoint circuit and using two sets of differential separation filters [16]. As a result, our improved design allows independent detection of chest and abdominal RM signals. In our system, the constitution of the differential separation filter was the same as that described by Yama and Ueno [10], but we set the cutoff frequency at $0.5 \mathrm{~Hz}$. We also used a low-pass analog filter with a cutoff frequency of $40 \mathrm{~Hz}$ to eliminate high-frequency components after the separation filter. Thus, the resulting frequency band for detecting ECG signals ranged from 0.5 to $40 \mathrm{~Hz}$. Likewise, we used a high-pass analog filter with a cutoff frequency of $0.15 \mathrm{~Hz}$ to eliminate low-frequency components, thus the resulting frequency band for detecting RM signals ranged from 0.15 to $0.5 \mathrm{~Hz}$. Finally, we amplified filtered signals one thousand times (60 dB).

\section{Methods}

All experimental procedures were approved by the Human Life Ethics Committee of Tokyo Denki University. All subjects provided informed consent prior to participation in our experiments.

\subsection{Evaluation of baseline restoration time}

Bedridden patients requiring monitoring of vital signs at home have to change their body position periodically to prevent bedsores [17]. Stable measurement is required even after such changes in body position. We therefore instructed the test subjects to roll over in bed on cue. In such instances, we measured the time taken for restoration of the ECG signal to baseline. One subject wearing commercially available nightwear made of cotton with a thickness of $0.36 \mathrm{~mm}$ participated in this experiment. Electrodes were placed under a bed sheet with a thickness of $0.33 \mathrm{~mm}$. Two patterns of signals were then measured on the initial circuit; one with a conventional voltage follower circuit, and the other with the proposed voltage follower circuit with a bootstrap.

The subject was first asked to lie on the bed in a supine position, and then changed the body position to a lateral position upon instruction. The subject was asked to remain in that position until baseline was restored. The subject then returned to the supine position upon instruction. Defining these procedures as a single set of experiment, ten sets were then carried out. The baseline restoration time was defined as the time taken for the ECG signal to return to $\pm 1 \mathrm{~V}$ (or lower) of the steady-state baseline level.

\subsection{Simultaneous measurements of chest and abdominal RM signals}

Although the normal respiration rate of adults is approximately 15 to $20 \mathrm{bpm}$ [18], it is necessary for the system to be applicable to a wider range of respiration rates. We therefore evaluated the proposed system in its ability to measure RM signals by conducting an experiment in one subject. First, the signals measured using a respiration transducer (BN-RESP-XDCR, BIOPAC Systems) and a breathing amplifier (RSP100C, BIOPAC Systems) were used as reference signals. The subject was asked to wear the respiration transducer on his chest and abdomen while lying on the bed in a supine position. The individual's clothing was the same as that described in Section 3.1. To vary respiration rate, the subject was instructed to breath in synchrony with a metronome. The subject was also asked to hold his breath for $10 \mathrm{~s}$ upon in- 
struction. The respiratory rate per minute was then calculated based on the detected signals.

\subsection{Overnight measurements}

Next, we evaluated our proposed system by conducting an experiment during sleep in seven healthy male subjects. The physical data of the seven subjects are shown in Table 1. A commercially available ECG amplifier (BA1104-CC, TEAC Instruments), telemeter (TU-4, Teac Instruments; A/D: $312.5 \mathrm{GHz}, 16$ bit, transmission frequency band: $315-322 \mathrm{~Hz}$ ), and disposable electrodes (F-150S, Nihon Kohden) were used to record reference lead III ECG signals. Note that the reference signal for RM was the same as the one described in Section 3.2, but each subject was asked to wear the respiration transducer only on the abdomen because of the overlapping arrangement of the chest transducer and the upper electrode. Videos were recorded to verify the body movements of the participants. The clothing was the same as that described in Section 3.1 above. The arrangement of the electrodes was adjusted individually according to the physique of the subject.

The duration of the experiment was at least six hours, although we performed analysis using only the data for the first six hours. In this experiment, subjects were free to change body positions as they wished. The low-frequency components of the ECG often overlapped with the RM signals measured by our proposed system. To reduce the influence of the low-frequency components, we digitally applied an offline band-pass filter (IIR, $f_{c l}=$ $\left.0.15, f_{c 2}=0.5, \mathrm{Q}=0.707\right)$ twice to the $\mathrm{RM}$ signals. To equalize the analytical conditions, we also applied the same process to the reference RM signal.

\subsection{Analysis of ECG and RM signals}

We detected the $\mathrm{Q}$ wave, the $\mathrm{R}$ wave, and the peak and end of the $T$ wave $\left(T_{\text {top }}\right.$ and $\left.T_{\text {end }}\right)$ on the reference ECG and the capacitive ECG measured by our proposed system by pattern matching using the wavelet transform [19]. We calculated the QT interval using the detected $\mathrm{Q}$ wave and $\mathrm{T}_{\text {end. }}$. The capacitive ECGs having an $\mathrm{R}$ wave detected within $\pm 3 \mathrm{~ms}$ of the $\mathrm{R}$ wave on the reference ECGs were considered to be synchronized. Further, we performed Bazett compensation on the QT intervals obtained from the reference and our proposed system, as the QT interval is known to depend on the R-R interval [20].

We used automatic detection software proposed by Yamagishi et al. [21] to detect RM signals. This software uses threshold processing. If the starting point of one cycle of an individual's RM measured by our proposed system was included in the interval of

Table 1 Age and physical constitution of the subjects who participated in the overnight experiment.

\begin{tabular}{ccccc}
\hline \hline subject & age [yrs] & height $[\mathrm{m}]$ & weight $[\mathbf{k g}]$ & BMI $\left[\mathbf{k g} / \mathbf{m}^{2}\right]$ \\
\hline$\# 1$ & 23 & 1.75 & 65.0 & 21.2 \\
$\# 2$ & 22 & 1.73 & 71.0 & 23.7 \\
$\# 3$ & 20 & 1.60 & 55.0 & 21.5 \\
$\# 4$ & 21 & 1.87 & 73.0 & 20.9 \\
$\# 5$ & 20 & 1.73 & 60.0 & 20.1 \\
$\# 6$ & 22 & 1.67 & 70.0 & 25.1 \\
$\# 7$ & 20 & 1.72 & 78.8 & 26.6 \\
\hline \hline
\end{tabular}

the corresponding cycle of the reference RM, the RM from our proposed system was deemed to be correctly detected.

Finally, we calculated the sensitivity and accuracy using Equations (2) and (3) for both ECG and RM signals [22]. In these equations, TP represents the number of target events ( $R$ wave, $T$ wave, and RM) correctly detected in each output signal of our system, FN is the number of target events undetected by our system, and FP is the number of target events falsely detected by our system. Since there are no countable valid events corresponding to true negative (TN), TN was set at zero in calculating accuracy [22].

$$
\begin{aligned}
& \text { Sensitivity }=\frac{T P}{\boldsymbol{T P}+\boldsymbol{F N}} \times 100 \\
& \text { Accuracy }=\frac{\boldsymbol{T P}+\boldsymbol{T N}}{\boldsymbol{T P}+\boldsymbol{T N}+\boldsymbol{F P}+\boldsymbol{F N}} \times 100
\end{aligned}
$$

\section{Results}

\subsection{Restoration time of ECG signals}

ECG and RM signals were obtained while the subject was in the supine position, as well as in the left and right lateral positions. Figure 4 shows examples of the waveforms representing restoration of ECG signal to baseline from the time of occurrence of body movement. The average time for restoration to baseline was $3.19 \mathrm{~s}$ when a conventional voltage follower was used as the front-end circuit and $2.83 \mathrm{~s}$ when the voltage follower with a bootstrap was used. The restoration time was effectively reduced by $0.36 \mathrm{~s}$ on average.

As shown in Fig. 4, there were some instances in which burst noise contaminated the ECG waveform during the period of recovery when a conventional voltage follower was used. There were also cases in which a relatively long time was required to resume observation of the ECG signals. Our method made it possible to visually confirm the ECG signal from the time when restoration to baseline started after saturation. We therefore confirmed that the voltage follower with a bootstrap contributed to reducing the time required for restoration of ECG signal to base-

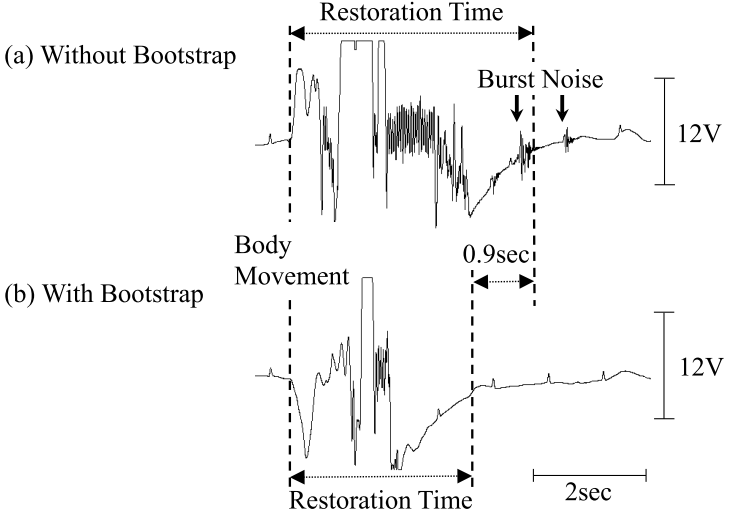

Fig. 4 Examples of measurement of time taken for ECG signal to restore to baseline: (a) wave pattern of ECG recorded with a front-end voltage follower; (b) wave pattern of ECG recorded with the proposed front-end voltage follower with a bootstrap. Dotted lines indicate the restoration phase from saturation. The baseline restoration time was shortened in (b). Arrows in (a) show the parts in which ECG cannot be observed without noise elimination. 
line following body movements.

\subsection{Evaluation of RM measurements}

We calculated the correlation between the respiration rates measured by a respiration transducer and the rate measured by the proposed system from both chest and abdomen signals. A high correlation coefficient of 0.98 was obtained for both RM signals. Figure 5 shows RM signal recordings, including a segment in which the participant held his breath. From the figure, we observe that RM signals obtained by the proposed system synchronized with the reference data. In the segment in which the participant held his breath, the amplitudes of signals from the proposed system were reduced compared with the reference data, indicating that the proposed system is indeed capable of responding to a substantial change in respiration rate. Portions in which respiration rates did not match were attributed to slight movements of the body. These errors are attributable to leg and other movements, as the subjects were awake during the experiment.

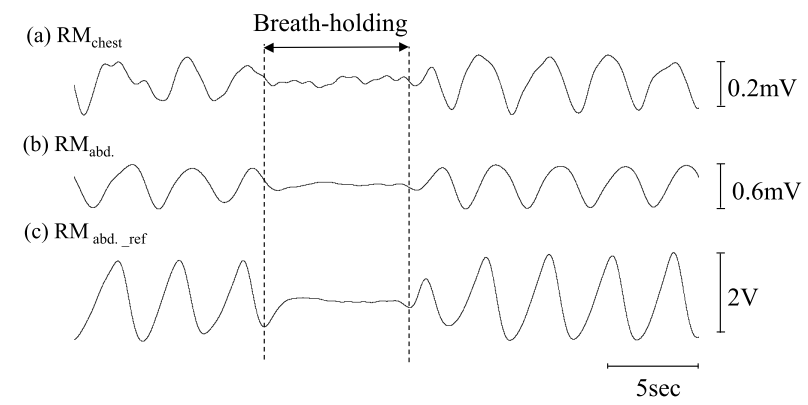

Fig. 5 Recording of RM signals while a participant held his breath, showing (a) chest movements $\left(\mathrm{RM}_{\text {chest }}\right)$ and (b) abdomen movements $\left(\mathrm{RM}_{\mathrm{abd} .}\right)$ obtained by the proposed system, as well as (c) abdomen movements ( $\left.\mathrm{RM}_{\text {abd_ref }}\right)$ obtained by a commercial belt-type sensor. Cessation of movement can be seen as relatively flat segment during period of holding one's breath.

\subsection{Sensitivity and accuracy of ECG and RM signal mea- surements}

Figure 6(a) shows an example of measurements obtained during sleep for Subject \#6. Body movement segments resembling the waveforms shown in the figure also existed in all subjects, but the frequencies and durations differed substantially from one subject to another. Figure 6(b) shows that the proposed system was able to measure the $\mathrm{R}$ and $\mathrm{T}$ waves during a stable segment. ECG signals similar to the waveforms shown in this figure were confirmed in all subjects.

The sensitivity and accuracy of each signal for each of the seven subjects are shown in Table 2. As noted above, the target analysis timeframe was the first six-hour period, but this target period was set at four hours for Subject \#4, because the reference ECG signal was not available for the last two hours. The average accuracy was greater than $90 \%$ for $\mathrm{R}$ and $\mathrm{T}$ waves. The number of falsely detected target events in the output signal (FP) for $\mathrm{T}$ wave was almost zero, because the sensitivity equals the accuracy for all subjects. Finally, the accuracy of RM ranged from $63 \%$ to $81 \%$.

\section{Discussion}

\subsection{Evaluation of overnight experiments}

The number of undetected target events (FN) was the main factor that degraded ECG accuracy. In some subjects, signals with numerous FNs existed in two types of segments: (1) segments in which fluctuation of the baseline was so drastic that visual verification was also not possible; and (2) segments in which the target event was undetected even though it seemed to synchronize with the reference. In segments (2), the signals were attenuated significantly to approximately $0.1 \mathrm{mV}$, whereas the typical amplitude of the ECG signal from the proposed system was approximately 0.8 to $1.5 \mathrm{mV}$. Any ECG signals with extremely low amplitudes that could not be detected by the analysis program were regarded as undetected (counted as FN) [19]. This extremely low voltage may be caused by overlapping electrodes or reduced coupling area due to repeated body movements, such as rolling over.

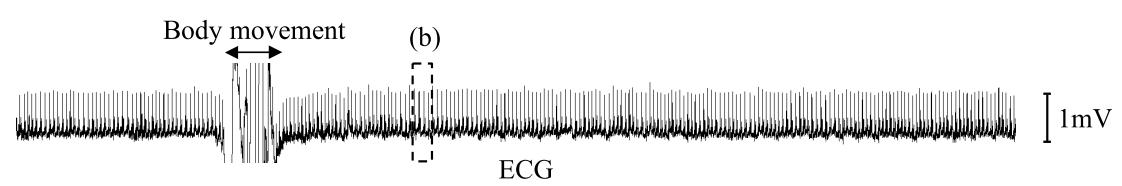

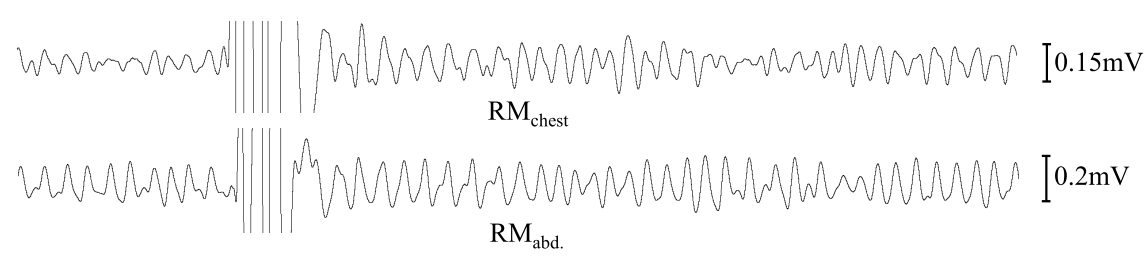

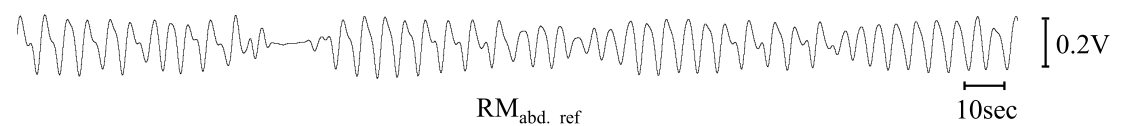

(a)

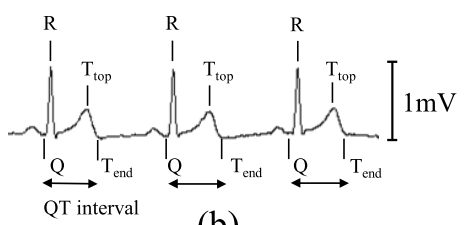

(b)

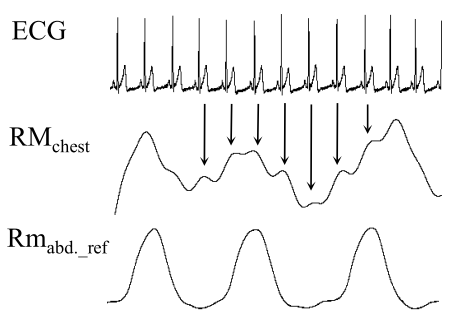

(c)

Fig. 6 Results of overnight measurements for Subject \#6. (a) Recordings of ECG and RM signals accompanying postural change. Due to this change, ECG and RM signals obtained by our system were undetectable for 10-20 s. (b) Enlarged waveforms of ECG signals for the segment enclosed by dotted line in (a), after analysis was performed by the automatic detection software. Overall, detected Q waves, $\mathrm{R}$ waves, and the peak and end of the $\mathrm{T}$ waves $\left(\mathrm{T}_{\text {top }}\right.$ and $\mathrm{T}_{\text {end }}$ ) are shown. Automatically calculated QT intervals are also indicated. (c) Example of RM $\mathrm{M}_{\text {chest }}$ recording accompanied by overlapped $\mathrm{T}$ waves. 
Table 2 Sensitivity and accuracy of ECG and RM signal measurements in seven subjects. In each subject, R wave and $\mathrm{T}$ wave of ECG, and chest and abdominal RMs were calculated independently.

\begin{tabular}{l|cc|cc|cc|cc}
\hline \multirow{2}{*}{ subject } & \multicolumn{4}{|c|}{ sensitivity[\%] } & \multicolumn{3}{c}{ accuracy[\%] } \\
\cline { 2 - 10 } & \multicolumn{2}{|c|}{ ECG } & \multicolumn{2}{c|}{ RM } & \multicolumn{2}{c}{ ECG } & \multicolumn{2}{c}{ RM } \\
\cline { 2 - 10 } & R wave & T wave & chest & abd. & R wave & T wave & chest & abd. \\
\hline$\# 1$ & 96.5 & 96.5 & 69.5 & 80.8 & 96.2 & 96.2 & 66.8 & 78.6 \\
$\# 2$ & 96.3 & 97.5 & 78.3 & 81.0 & 96.0 & 97.5 & 73.9 & 77.2 \\
$\# 3$ & 83.8 & 87.6 & 72.8 & 74.4 & 83.8 & 87.6 & 70.9 & 72.3 \\
$\# 4$ & 94.4 & 97.3 & 89.4 & 90.1 & 94.4 & 97.3 & 79.7 & 81.3 \\
$\# 5$ & 92.2 & 92.9 & 69.7 & 72.6 & 92.1 & 92.8 & 66.2 & 68.9 \\
$\# 6$ & 97.1 & 95.4 & 82.9 & 83.1 & 96.1 & 95.4 & 75.4 & 75.2 \\
A7 & 91.8 & 94.4 & 69.9 & 71.7 & 91.8 & 94.4 & 63.5 & 64.7 \\
\hline \hline
\end{tabular}

*For Subject \#4, four-hour data were analyzed, because reference ECG was unmeasurable during the experiment.

Once the coupling area was reduced, the capacitance value from the capacitive coupling also decreased, resulting in attenuation of the signals. More frequent body movements and position changes such as rolling over occurred in Subjects \#3 and \#5. To avoid shifting of electrodes away from the body and losing capacitive coupling to the body, integrating electrodes may be required in the future. In addition, the signal also depends on the distance between the body and the electrodes. This distance is approximately the same as the total thickness of the bed sheet and nightwear. As noted above, the thicknesses of the bed sheet and nightwear were $0.33 \mathrm{~mm}$ and $0.36 \mathrm{~mm}$, respectively. Therefore, the total thickness of insulation was $0.69 \mathrm{~mm}$. Although the thickness did not seem to influence our results, there may be an upper limit of thickness that maintains good signal acquisition.

The average accuracies of both chest and abdominal RMs were relatively low (70.9 and 74.0\%). One possible reason for the low sensitivity is that the position of electrodes shifted causing a reduction in RM signal amplitude. Further, the baseline restoration time of the RM signals was longer than that of the ECG results, as shown in Fig. 6(a). Therefore, the large variations in accuracy and sensitivity between subjects are attributable to individual differences in the frequency of body movements.

Another reason for the relatively low accuracy is that low-frequency components of ECG signals may have overlapped with the RM signal, as shown in Fig. 6(c). This may be caused by subjects sweating as the sleeping duration increased. The RM signal is captured based on a change in distance between the body and electrodes, but the RM signal also depends on electric charge accumulated in the coupling area. Coupling between the body and electrodes is probably improved by the presence of sweat, which then reduces the impedance of the coupling. As a result, larger ECG voltage overlapped and made RM signal detection more difficult. It is possible to reduce the frequency of sweat-induced detection error by increasing the order of the separation filters in the proposed system, a task that we shall undertake in our future work.

\subsection{Comparison of $\mathrm{QT}_{\mathrm{c}}$ interval between the proposed sys- tem and commercial system}

We investigated the correlation between the compensated QT time $\left(\mathrm{QT}_{\mathrm{c}}\right.$ intervals) obtained by the reference and the proposed systems during a 20-minute stable segment for all subjects using a Bland-Altman plot. Results for Subject \#9 are shown in Fig. 7. The correlation coefficient for this subject was 0.90 , showing a high degree of accuracy. Although $\mathrm{QT}_{\mathrm{c}}$ interval is known to vary depending on gender, age and race, when normal $\mathrm{QT}_{\mathrm{c}}$ interval is defined as $440 \mathrm{~ms}$ or less [23, 24], our data were all within the normal range. Further, the estimated error of $\mathrm{QT}_{\mathrm{c}}$ interval of the proposed system is within $\pm 6.4 \mathrm{~ms}$, by subtracting fixed bias of $-9 \mathrm{~ms}$ from the current value for $\mathrm{QT}_{\mathrm{c}}$ interval. Therefore, detection of long QT syndrome may be possible by monitoring ECG using the proposed system.

\subsection{Possibility of breathing effort detection}

In obstructive sleep apnea, chest and abdominal movements still exist even though no air flows through the airway, but these movements are known to be in opposite phases [25]. Three academic societies have jointly developed guidelines for home-monitoring of sleep apnea [26]. The proposed system may be classified as a type 3 device when used in conjunction with a pulse oximeter, because the proposed system is able to measure chest and abdominal RM signals independently. We therefore performed an experiment in which a subject was instructed to simulate obstructed respiration at an arbitrary time. The observed RM waveforms, including the individual's breathing effort (BE), are shown in Fig. 8. In the first one-third of the recording, the chest and abdominal RM waveforms were in the same phase. Both RMs obtained by the proposed system also synchronized with the reference RMs. In the second one-third, the trough of the chest RM corresponded to the peak of the abdominal RM during simulated obstructive respiration, indicating that the RM signals were in opposite phase. In the last one-third, the signals returned to being in phase with one another after resuming normal respiration.

These results suggest that the proposed system is capable of detecting phase changes. Luis JA et al. [27] proposed a system that uses capacitive sensing principles of LC oscillators. A com- 


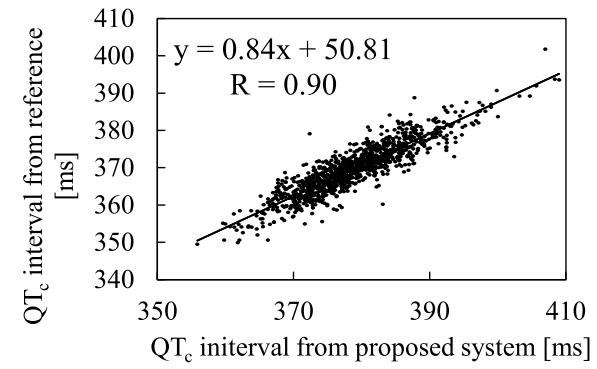

(a) Relationship ( $\mathrm{QT}_{\mathrm{c}}$ interval)

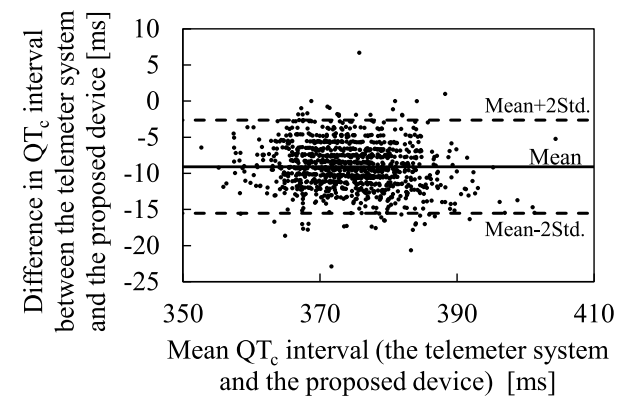

(b) Bland-Altman plots $\left(\mathrm{QT}_{\mathrm{c}}\right.$ interval)

Fig. 7 (a) Relationship between $\mathrm{QT}_{\mathrm{c}}$ interval obtained from a telemeter and proposed system. (b) Bland-Altman plots between $\mathrm{QT}_{\mathrm{c}}$ interval from the telemeter and proposed system (Subject \#7). (a) and (b) were calculated from a stable segment of 20 minutes.

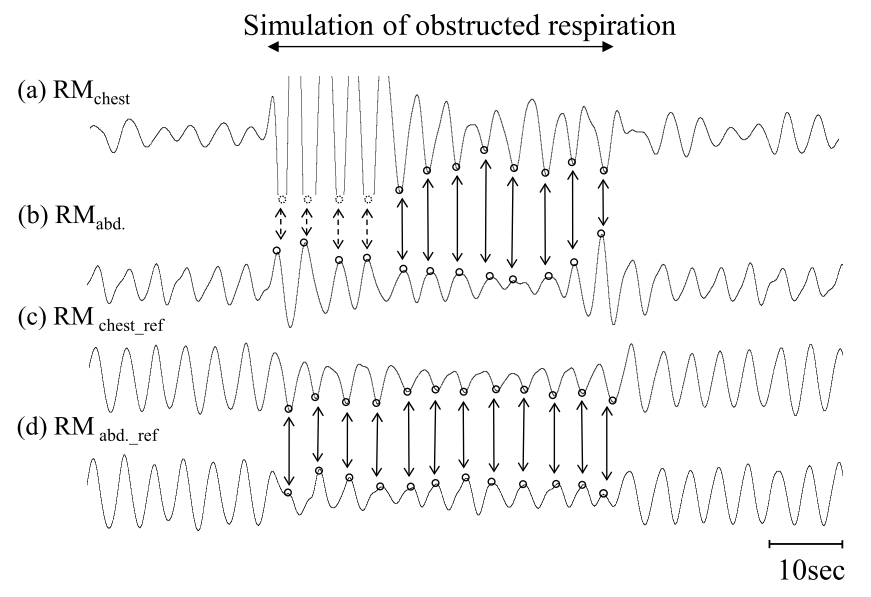

Fig. 8 Recordings of RM accompanying breathing effort during simulation of obstructed respiration. (a) Chest and (b) abdomen movements obtained by the proposed system, and (c) chest and (d) abdomen movements obtained by a commercial belttype sensors are shown.

bination of our proposed system and their system may be beneficial to improve the accuracy of RM detection.

\section{Conclusion}

In this study, we improved a measuring device for ECG and RM signals, with the aim to monitor vital signs in adults in the home setting. We evaluated the performance of our system by conducting overnight experiments. The results indicated that simultaneous measurements of ECG signals, including T wave and QT in- terval measures, and respiratory signals were possible when the individuals maintained ordinary body positions such as supine position and left and right lateral positions. Furthermore, the time required for ECG signal to return to baseline after body movements was successfully reduced. As for ECG measurements, our six-hour overnight experiments revealed that the average sensitivity and accuracy for $\mathrm{R}$ and $\mathrm{T}$ waves were greater than $90 \%$ in seven subjects. The correlation coefficient for $\mathrm{QT}_{\mathrm{c}}$ interval between the reference system and our proposed system was 0.90 . Therefore, we expect the proposed system to be applicable to monitoring vital signs. As for the RM measurements, although a high correlation in respiration rate of 0.98 was achieved for both chest and abdominal RM signals, accuracy was only approximately $70 \%$, suggesting a need for improvement of the RM detection circuit.

\section{Conflict of Interest}

The authors declare no conflicts of interest regarding this manuscript, nor do they have any relationship with companies or commercial organizations based on the definition of Japanese Society of Medical and Biological Engineering.

\section{Acknowledgement}

This research was supported by the Strategic Research Foundation Grant-aided Project for Private Universities from Ministry of Education, Culture, Sport, Science and Technology, Japan, 20152017 (S1512001).

\section{References}

1. United Nations: World population prospects the2012 revision. $<$ https://esa.un.org/unpd/wpp/publications/Files/WPP2012_ HIGHLIGHTS.pdf $>$ [accessed on May 17, 2016]

2. Motomura K, Arai I: Development of a heart rate monitor used a $24 \mathrm{GHz}$ microwave Doppler sensor. Jpn J Med Ele Biol Eng. 35(3), pp. 265-271, 1997. In Japanese.

3. Kranjec J, Beguš S, Drnovšek J, Geršak G: Novel methods for noncontact heart rate measurement: A feasibility study. IEEE Trans Instrum Meas. 63(4), pp. 838-847, 2013.

4. Hoshiba K, Hirata S, Hachiya H: Simultaneous measurement of respiration and heart rate using airborne ultrasound. Proc Symp Ultrasonic Ele. 36. USE. Japan, 3P2-2-1-2, 2015.

5. Saitoh Y, Hori J, Kiryu T: Development of unaware detection system for respiration and heart beats using a magnetic sensor. Jpn J Med Ele Biol Eng. 38(2), pp. 102-110, 2000. In Japanese.

6. Ishijima M: Cardiopulmonary monitoring by textile electrodes without subject-awareness of being monitored. Med Biol Eng Comput. 35(6), pp. 685-690, 1997.

7. Park SB, Noh YS, Park SJ, Yoon HR: An improved algorithm for respiration signal extraction from electrocardiogram measured by conductive textile electrodes using instantaneous frequency estimation. Med Biol Eng Comput. 46, pp. 147-158, 2008.

8. Lee HJ, Hwang SH, Yoon HN, Lee WK, Park KS: Heart rate variability monitoring during sleep based on capacitively coupled textile electrodes on a bed. Sensors. 15(5), pp. 11295-11311, 2015.

9. Ueno A, Yama Y: Unconstrained monitoring of ECG and respiratory variation in infants with underwear during sleep using a bedsheet electrode unit. Proc 30th Ann Int Conf IEEE EMBS. pp. 2329-2332, 2008.

10. Yama Y, Ueno A: Unrestrained facile measurement of narrow-band ECG and respiratory variation in infants with a capaci- 
tive sheet-type sensor. Trans Jpn Soc Med Biol Eng. 47(1), pp. 42-50, 2009. In Japanese.

11. Peltokangas M, Verho J, Vehkaoja A: Night-time EKG and HRV monitoring with bed sheet integrated textile electrodes. IEEE Trans Inf Technol Biomed. 16(5), pp. 935-942, 2012.

12. Fukuyama Y, Suzuki R, Takayama S, Ueno A: Multi-layered fabric electrode for movement artifact reduction in capacitive ECG measurement. Proc 35th Ann Int Conf IEEE EMBC. pp. 555558, 2013.

13. Takayama S, Ueno A: Mitigation of movement artifact in capacitive in-vehicle electrocardiogram measurement. Proc 12th Int Conf u-Healthcare. pp. 23, 2015.

14. Ueno A, Shiogai Y, Ishiyama Y: A primary study of indirect ECG monitor embedded in a bed for home health care. IEEJ Trans Ele Inf Syst. 127(10), pp. 1792-1799, 2007. In Japanese.

15. Shirato Y: All about analogue IC: Illustration from OP-amp to switched capacitor. Tokyo Denki University Press, Japan, pp. 149-151, 1986. In Japanese.

16. Ueno A: Development of measurement technique for simultaneous detection of two electrocardiograms and two breathing effort signals using sheeted fabric electrode. In: N. T. S. Co., Ltd. ed. Personal Healthcare: Cutting-edge electronics for realizing ubiquitous and wearable medicine. N. T. S. Co., Ltd. Press, Japan, pp. 49-56, 2013. In Japanese.

17. Guidelines revision committee of the Japanese Society of Pressure Ulcers: Guidelines for the prevention and management of bedsore, 3rd ed. Jpn J PU. 14(2), pp. 165-226, 2012. In Japanese.

18. Maruyama H: Risk management: Vital signs. Rigakuryoho Kagaku. 20(1), pp. 53-58, 2005. In Japanese.

19. Ohmuta T, Iimura K, Mitsui K, Shibata N: Development of a QT measurement method by applying wavelet transform analysis and its correlation with QaT measurement. Jpn J Electrocardiol. 26(6), pp. 803-809, 2006. In Japanese.

20. Bazett HC: An analysis of the time-relations of electrocardiograms. Heart. 7, pp. 353-370, 1920.

21. Yamagishi S, Takano M, Ueno A, Fukuoka Y: A study on breath detection algorithm for capacitive in-bed cardiorespiratory monitor. IECE technical report. 115(513), MBE2015-103, pp. 5-8, 2016. In Japanese.

22. Lee JS, Heo J, Lee WK, Lim YG, Kim YH, Park KS: Flexible capacitive electrodes for minimizing motion artifacts in ambulatory electrocardiograms. Sensors. 14(8), pp. 14732-14743, 2014.

23. Grandinetti A, Seifried S, Mor J, Chang HK, Theriault AG: Prevalence and risk factors for prolonged QTc in a multiethnic. Clin Biochem. 38(2), pp. 116-122, 2005.

24. Akiyama T: IX. Assessment of cardiac function and prediction of prognosis by QT interval. Jpn J Electrocardiol. 33(3), pp. 223247, 2013. In Japanese.

25. Murata A: Diagnosis and treatment of sleep apnea syndrome: Sleep apnea syndrome triggers life-threatening disorders during sleep. Nihon Ika Daigaku Igakkai Zasshi. 3(2), pp. 96-101, 2007. In Japanese.

26. Flemons WW, Littner MR, Rowley JA, Gay P, Anderson WM, Hudgel DW, McEvoy RD, Loube DI: Home diagnosis of sleep apnea: A systematic review of the literature: An evidence review cosponsored by the American academy of sleep medicine, the American college of chest physicians, and the American thoracic society. Chest. 124(4), pp. 1543-1579, 2003.

27. Luis JA, Roa Romeo LM, Gómez-Galán JA, Hernández DN, Estudillo-Valderrama MÁ, Barbarov-Rostán G, Rubia-Marcos C: Design and implementation of a smart sensor for respiratory rate monitoring. Sensors. 14(2), pp. 3019-3032, 2014.

\begin{abstract}
Mayuko TAKANO
Mayuko TAKANo received the B.S. degree in electrical and electronic engineering from Tokyo Denki University, Tokyo, Japan, in 2016. She is currently a graduate student in Master's program of Electrical and Electronic Engineering, Graduate School of Engineering, Tokyo Denki University, Tokyo, Japan. Her research interests include biomedical instrumentation and analog circuit. She received encouragement prizes from IEEJ Tokyo Branch and from the Society of Life Support Engineering, and poster award of Biomedical Engineering Symposium 2016.
\end{abstract}

\section{Shinsuke YAMAGishI}

Shinsuke YAmagishi received the BS degree in electrical engineering from Kogakuin University, Tokyo, Japan, in 2012. He is currently a graduate student in Master's program of Electrical and Electronic Engineering, Graduate School of Engineering, Kogakuin University, Tokyo, Japan. His research interests include biomedical instrumentation.

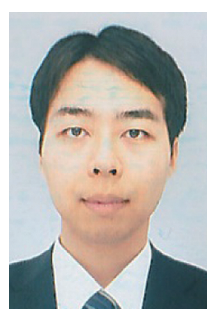

\section{Takao ОнмитА}

Takao ОнмитA received a bachelor's degree in precision mechanics from Tokyo Denki University in 2002, and completed the doctoral program at the same university (School of Advanced Engineering) in 2007 (D.Eng.).Researcher at AIST 2007, then researcher at Tokyo Denki University (Faculty of

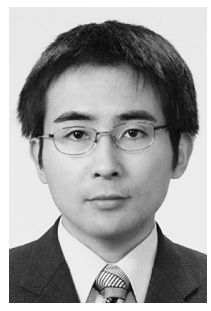
Engineering); Associate professor at Tokyo Denki University (the Research Center for Advanced Technologies) 2008, since 2013 associate professor at Suzuka University of Medical Science (Department of Clinical Engineering, Faculty of Medical Engineering).Specialties: ECG signal processing. Membership: JHRS, JSMBE, JSLST.

\section{Yutaka FuKuoKa}

Yutaka FuкuоKa received the BS, MS and PhD degrees in electrical engineering from Keio University, Yokohama, Japan, in 1987, 1989 and 1992, respectively. In 1992, he joined the Institute of Medical and Dental Engineering, Tokyo Medical and Dental University, Tokyo, Japan. He was a Vis-

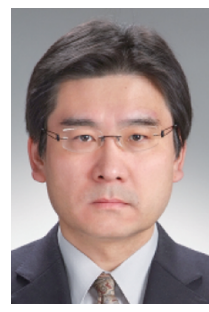
iting Scientist at Harvard-MIT Division of Health Science and Technology, Cambridge, MA, in 1999 and 2000. In 2012, he joined the Department of Electrical Engineering, Kogakuin University, where he is currently a Professor. His research interests include biosystems modeling, and systems biology. 


\section{Akinori Ueno}

Akinori UENo received the B.S. degree in electrical engineering in 1994, and the M.S. and Ph.D. degrees in biomedical engineering in 1996 and 1999, respectively from Keio University, Yokohama, Japan. He is currently a Professor of electrical and electronic engineering at Tokyo Denki University,

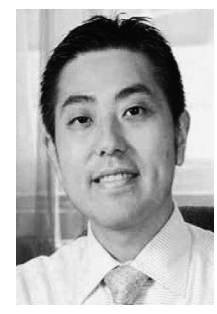
Tokyo, Japan, and held a position as Associate Professor of electronic and computer engineering at Tokyo Denki University, Saitama, Japan. He was a Visiting Scholar at bioengineering department of University of California-San Diego, La Jolla, CA, from April 2013 to March 2014. His research interests include biomedical instrumentation and intelligent human-machine interfaces. Dr. Ueno is the recipient of several research awards from the Japan Society of Medical Electronics and Biological Engineering, the Society of Life Support Engineering and the Society of Instrument and Control Engineers. 